\title{
Binary consistent choice on pairs and a generalization of Konig's infinity lemma
}

by

\section{Paul E. Howard (Ypsilanti, Mich.)}

Abstract. In this paper we answer in the affirmative the question of Cowan: Does $T_{2} \rightarrow \mathrm{BPI}$ ? where $T_{2}$ is Cowan's generalization of Konig's infinity lemma restricted to trees of order 2 . We also give a negative answer to the question: Does $F_{2} \rightarrow B P I$ ? where $F_{2}$ is a principle involving binary consistent choice on pairs and BPI is the Boolean prime ideal theorem.

1. In [2] R. Cowan generalizes Konig's infinity lemma. We begin by describing that generalization. For convenience we give the following definitions from [2].

A tree is a connected undirected graph without circuts one of whose vertices is designated as the origin. The number of vertices on the unique path connecting a vertex $v$ with the origin is the level of $v, l(v)$. (Thus the set of vertices of a tree can be decomposed into an at most denumerable set of levels.) A vertex $v^{\prime}$ is a successor of a vertex $v$ if $v$ and $v^{\prime}$ are connected by an edge and $l\left(v^{\prime}\right)=l(v)+1$.

A tree is finite if its set of vertices is finite and locally finite if each vertex has only finitely many successors. A branch in a tree is a maximal path beginning at the origin. If $v$ and $v^{\prime}$ are on the same branch, then $v^{\prime}$ dominates $v$ if $l\left(v^{\prime}\right) \geqslant l(v)$. Konig's lemma states that any infinite locally finite tree has an infinite branch.

Let $T$ be a collection of locally finite trees (not necessarily pairwise disjoint). By a vertex or a level of $T$, we mean a vertex or a level of some tree in $T$. Also if $v$ and $v^{\prime}$ are vertices of $T$, then $v^{\prime}$ dominates $v$ in $T$ if $v^{\prime}$ dominates $v$ in some tree in $T$. Let $S$ be a set of vertices of $T . S$ pierces a level $l$ of $T$ if $|S \cap l|=1$. (For any set $A,|A|$ denotes the cardinal number of $A$.) $S$ is consistent if for every $v, v^{\prime}$ in $S$ there is a $v^{\prime \prime}$ which dominates them both in $T$ We can now state R. Cowan's generalization of Konig's lemma.

THEOREM 1.1. Let $T$ be a collection of locally finite trees such that for any finite set of levels of $T$, there is a consistent set of vertices piercing those levels. Then there is a consistent set of vertices piercing the entire set of levels of $T$.

Let $\left\{A_{i}\right\}_{i \in I}$ be a collection of sets and $R$ a symmetric binary relation on $\bigcup A_{i}$. A choice function $f$ for $\left\{A_{i}\right\}_{i \in I}$ js- $R$-consistent if $f(i) R f(j)$ for all $i, j$ in $I$ 
with $i \neq j$. We will also consider the following theorem of Łos and RyllNardzewski:

THeOREM 1.2. Let $\left\{A_{i}\right\}_{i \in I}$ be a collection of finite sets and $R$ a symmetric binary relation on $\bigcup_{i \in I} A_{i}$. Suppose that for every finite $W \subseteq I$, there is an $R$ consistent choice function for $\left\{A_{i}\right\}_{i \in W}$. Then there is an R-consistent choice function for $\left\{A_{i}\right\}_{i \in I}$.

It is known [1] and [6] that both of the above theorems are equivalent to the Boolean prime ideal theorem (BPI).

We now define the order of a collection of locally finite trees $T(o(T))$ to be the least cardinal $f$ such that no tree in $T$ contains a vertex with more than $\mathfrak{f}$ successors and let $T_{n}$ be the statement of Theorem 1.1 only for $T$ with $o(T)=n, n$ a positive integer. Also let $F_{n}$ denote the statement of Theorem 1.2 where it is required that $\left|A_{i}\right| \leqslant n$ for all $i \in I$.

In [2] it is shown that $T_{n} \rightarrow F_{n} \rightarrow$ BPI for any integer $n \geqslant 3$ and the question is posed: Does $T_{2} \rightarrow \mathrm{BPI}$ or $F_{2} \rightarrow \mathrm{BPI}$ ? The purpose of this paper is to answer both questions. In Section 2 we give a proof of BPI from $T_{2}$ and in Section 3 we construct a Fraenkel-Mostowski model of ZFU (ZermeloFraenkel set theory weakened to permit the existence of urelements) in which $F_{2}$ is true and BPI is false. Actually, in the model constructed, the axiom of choice for sets of three element sets fails, so it appears that $F_{2}$ is considerably weaker than BPI.

2. In this section we prove:

THEOREM 2.1. $T_{2}$ implies the compactness theorem for propositional logic.

Proof. Let $K$ be an infinite set of propositional formulas such that every finite subset of $K$ is satisfiable. Let $P$ be the set of propositional variables occurring in $K$.

Lemma. If $P_{0}$ is any finite subset of $P$ and $K\left(P_{0}\right)$ is $\{x \in K$ : all the propositional variables in $x$ are in $\left.P_{0}\right\}$ then $K\left(P_{0}\right)$ is satisfiable.

Proof. If $K\left(P_{0}\right)$ is not satisfiable, then for each truth assignment $\sigma$ for the variables in $P_{0}$ there is an $x_{\sigma}$ in $K\left(P_{0}\right)$ such that $\sigma\left(x_{\sigma}\right)=F$. Therefore $\left\{x_{\sigma}: \sigma\right.$ is a truth assignment for $\left.P_{0}\right\}$ is a finite, nonsatisfiable set. This proves the lemma.

Now to complete the proof of Theorem 2.1 we follow the proof of Theorem 7 in [2]. Suppose that $W$ is a finite subset of $P$. A sequence of subsets of $W, W_{1}, W_{2}, \ldots, W_{k}$ is a $W$-tower if $W_{1}$ is a singleton, $W_{k}=W$ and $W_{i+1}=W_{i} \cup\{x\}$ for some $x, i=1,2, \ldots, k-1$. For each $W$-tower we form a tree as follows: The origin is $\phi$, level $i+1$ is

$F_{W_{i}}=\left\{\sigma: \sigma\right.$ is a truth assignment for $W_{i}$ such that $\sigma(x)=T$ for all $\left.x \in K_{W_{i}}\right\}$. $\sigma \in F_{W_{i}}$ is connected to $\sigma \mid W_{i-1}$ which belongs to $F_{W_{i-1}}$. Each vertex has at most two successors, therefore if $T$ is the set of all such trees, $o(T)=2$. If
$F_{W_{1}}, F_{W_{2}}, \ldots, F_{W_{m}}$ is any finite set of levels of $T$, then $V=\bigcup_{i=1}^{m} W_{i}$ is a finite subset of $P$. By the lemma, therefore, $F_{V} \neq \varnothing$. Suppose $\sigma \in F_{V}$, then $\left\{\sigma\left|W_{1}, \sigma\right| W_{2}, \ldots, \sigma \mid W_{m}\right\}$ is a consistent set of vertices since $\sigma$ dominates $\sigma \mid W_{i}$ in all the trees formed from $V$-towers containing $W_{i}$.

Further $\left\{\sigma\left|W_{1}, \sigma\right| W_{2}, \ldots, \sigma \mid W_{m}\right\}$ pierces each $F_{W_{i}}, \quad i=1,2, \ldots, m$. Therefore by $T_{2}$ there is a consistent set $F$ such that

$$
\left|F \cap F_{W}\right|=1 \text { for all finite } W \subseteq P .
$$

Since any two truth assignments in $F$ are restrictions of the same truth assignment, $F$ uniquely determines a truth assignment for $P$ which satisfies $K$. This completes the proof of the theorem.

Since the compactness theorem for propositional logic implies BPI [1], we have

Theorem 2.2. $T_{2}$ implies BPI.

3. In this section we prove that the implication $F_{2} \rightarrow \mathrm{BPI}$ does not hold by constructing a Fraenkel-Mostowski model in which $F_{2}$ is true and BPI fails.

Given a model $M^{\prime}$ of $\mathrm{ZFU}+\mathrm{AC}$ which has $U$ as its set of urelements, a permutation model $M$ of $\mathrm{ZFU}$ is determined by a group $G$ of permutations of $U$ and a filter of subgroups $\Gamma$ of $G$ which satisfies

and

$$
(\forall a \in U)(\exists H \in \Gamma)(\forall \varphi \in H)(\varphi(a)=a)
$$

$$
(\forall \varphi \in G)(\forall H \in \Gamma)\left(\varphi H \varphi^{-1} \in \Gamma\right) \text {. }
$$

Each permutation of $U$ extends uniquely to a permutation of $M^{\prime}$ by $\epsilon$-induction and for any $\varphi \in G$, we identify $\varphi$ with its extension.

If $H$ is a subgroup of $G$ and $x \in M^{\prime}$ and $(\forall \varphi \in H)(\varphi(x)=x)$ we say $H$ fixes $x$. If it is also the case that $(\forall \varphi \in H)(\forall y \in x)(\varphi(y)=y)$ we say that $H$ fixes $x$ pointwise. The permutation model $M$ determined by $U, G$ and $\Gamma$ consists of all those $x \in M^{\prime}$ such that for every $y$ in the transitive closure of $x$, there is some $H$ in $\Gamma$ such that $H$ fixes $y$. We refer the reader to $[4$, p.46] for the proof that $M$ is a model of $\mathrm{ZFU}$.

For our proof we assume that $M^{\prime}$ is a model of $\mathrm{ZFU}+\mathrm{AC}$ with a countable set of urelements $U$. We also assume for convenience that $U$ $=\bigcup_{i \in \omega} U_{i}$ where $U_{i} \cap U_{j}=\varnothing$ if $i \neq j$ and $U_{i}=\left\{a_{i}, b_{i}, c_{i}\right\}, i=0,1,2, \ldots$ For each $i \in \omega$, define $\eta_{i}: U_{i} \rightarrow U_{i}$ by $\eta_{i}\left(a_{i}\right)=b_{i}, \eta_{i}\left(b_{i}\right)=c_{i}$ and $\eta_{i}\left(c_{i}\right)=a_{i} . G$ is then defined to be the group of permutations

$$
G=\left\{\varphi: \varphi: \underset{\text { onto }}{\stackrel{1-1}{\rightarrow}} U \text { and }(\forall i \in \omega)\left(\varphi \mid U_{i}=\eta_{i} \text { or } \varphi \mid U_{i}=\eta_{i}^{2} \text { or } \varphi \mid U_{i}=1_{U_{i}}\right\}\right.
$$

where $1_{U_{i}}$ is the identity permutation on $U_{i}$. 
If $S$ is any finite subset of $\omega$ we define the subgroup $G_{S}$ of $G$ by

$$
G_{s}=\left\{\varphi \in G:(\forall i \in S)\left(\varphi \text { fixes } U_{i} \text { pointwise }\right)\right\}
$$

and the filter $\Gamma$ of subgroups of $G$ is

$$
\Gamma=\left\{G_{S}: S \text { is a finite subset of } \omega\right\} .
$$

"Lemma 1. $G$ is commutative.

This follows from the definition of $G$.

LeMma 2. For any $x \in M$, there is a smallest finite subset $S$ of $\omega$ such that $\left(\forall \varphi \in G_{S}\right)(\varphi(x)=x)$.

Proof. It suffices to show that the intersection of two subsets of $\omega$ satisfying the condition of the lemma is also such a subset. Suppose that $G_{S_{1}}$ and $G_{S_{2}}$ both fix $x$ and suppose that $\psi \in G_{S_{1} \cap S_{2}}$. To complete the proof we show that $\psi(x)=x$. Define $\varphi_{1} \in G$ and $\varphi_{2} \in G$ as follows:

$$
\begin{aligned}
& \varphi_{1}(t)= \begin{cases}\psi(t) & \text { if } t \in U_{i} \text { where } i \in S_{2}-S_{1}, \\
t & \text { otherwise, }\end{cases} \\
& \varphi_{2}(t)= \begin{cases}\psi(t) & \text { if } t \in U_{i}, \\
t & \text { otherwise } i \notin S_{2},\end{cases}
\end{aligned}
$$

Then we have $\varphi_{1} \in G_{S_{1}}, \varphi_{2} \in G_{S_{2}}$ and $\psi=\varphi_{1} \varphi_{2}$. Therefore $\psi(x)=\varphi_{1} \varphi_{2}(x)$ $=x$.

Defintrion. If $x \in M$ and $S$ is the smallest finite subset of $\omega$ such that $G_{S}$ fixes $x$, then $S$ is called the support of $x$.

The following lemma also follows from the definition of $G$ :

Lemma 3. For any $\varphi \in G, \varphi^{3}=1_{U}$.

For each $i \in \omega$ we define $\eta_{i}^{\prime}=\eta_{i} \cup 1_{U-v_{i}}$ and we note that $\eta_{i}^{\prime} \in G$.

THEOREM 3.1. BPI is false in $M$.

Proof. The set $X=\left\{U_{i}: i \in \omega\right\}$ has support $\phi$ and is therefore in $\boldsymbol{M}$. No choice function for $X$ is in $M$, for if $f$ is such a choice function with support $S$, we choose an integer $i \notin S$ and a $\varphi \in G_{S}$ such that $\varphi \mid U_{i}=\eta_{i}$. Without loss of generality assume that $f\left(U_{i}\right)=a_{i}$. Then $\varphi\left(U_{i}\right)=U_{i}$ but $\varphi\left(f\left(U_{i}\right)\right)=\varphi\left(a_{i}\right)=b_{i} \neq f\left(U_{i}\right)$, hence $\varphi$ does not fix $f$, a contradiction. Therefore the axiom of choice for sets of 3-element sets is false in the model and the theorem follows since BPI implies this form of AC.

Theorem 3.2. $F_{2}$ is true in $M$.

Proof. Let $A$ be any set of pairs in the model and $R$ a binary relation $(R \in M)$ such that the hypotheses of $F_{2}$ are satisfied. (I.e., for any finite subset $B$ of $A, B$ has an $R$-consistent choice function.) Let $S_{0} \subseteq \omega$ be the support of $\langle A, R\rangle$. For each $t \in A, \mathrm{OB}_{t}=\left\{\varphi(t): \varphi \in G_{S_{0}}\right\}$ is the orbit of $t$ under the group $G_{S_{0}}$ and we let $\mathrm{OB}$ be the set of orbits of elements of $A$ under the group $G_{S_{0}}$. (Then each $t \in A$ is in exactly one orbit and $\mathrm{OB}$ is well-ordered in $M$ since it is fixed pointwise by $G_{S_{0}}$.)

We apply $F_{2}$ in the model $M^{\prime}$ to get an $R$-consistent choice function $g$ for $A$. $g$ need not be in $M$, but we plan to modify $g$ to get an $r$-consistent choice function $f$ for $A$ which is in $M$.

For each $t=\{a, b\} \in A$, define $\sup (t)=S-S_{0}$, where $S$ is the support of $t$. For each finite $S \subseteq \omega$ such that $S \cap S_{0}=\varnothing$ define

$$
\operatorname{perm}(S)=\left\{\prod_{i \in S}\left(\eta_{i}^{\prime}\right)^{\Delta_{i}}: \Delta_{i} \in\{0,1,2\} \text { for all } i \in S\right\}
$$

If $t \in A$, we will write $\operatorname{perm}(t)$ for $\operatorname{perm}(\sup (t))$. Note that $|\operatorname{perm}(t)|=3^{|\sup (t)|}$ and is therefore an odd natural number. We also note that perm $(t)$ is a subgroup of $G_{S_{0}}$. In addition we have:

Lemma 4. If $t \in A$ and $t^{\prime} \in \mathrm{OB}_{t}$, then $t^{\prime}=\psi(t)$ for some $\psi \in \operatorname{perm}(t)$.

Proof. Suppose $t^{\prime} \in \mathrm{OB}_{t}$, then $t^{\prime}=\psi^{\prime}(t)$ for some $\psi^{\prime} \in G_{S_{0}}$. Define $\psi$ by

$$
\psi(x) \in \begin{cases}\psi^{\prime}(x) & \text { if } x \in U_{i} \text { for some } i \in \sup (t), \\ x & \text { otherwise. }\end{cases}
$$

Then $\psi \in \operatorname{perm}(t)$ and further since for all $x$ such that $x \in U_{i}$ for some $i \in \sup (t) \psi^{-1} \psi^{\prime}(x)=x$, we have $\psi^{-1} \psi^{\prime}(t)=t$ so that $\psi(t)=\psi^{\prime}(t)=t^{\prime}$. This completes the proof of the lemma.

Now suppose $t=\{a, b\} \in A$. We define

and

$$
\operatorname{perm}(t, a)=\{\psi \in \operatorname{perm}(t): g(\psi(t))=\psi(a)\}
$$

$$
\operatorname{perm}(t, b)=\{\psi \in \operatorname{perm}(t): g(\psi(t))=\psi(b)\} .
$$

Then $|\operatorname{perm}(t)|=|\operatorname{perm}(t, a)|+|\operatorname{perm}(t, b)|$ therefore since $|\operatorname{perm}(t)|$ is odd, $|\operatorname{perm}(t, a)| \neq|\operatorname{perm}(t, b)|$. We can therefore define

$$
f(t)=\left\{\begin{array}{l}
a \text { if }|\operatorname{perm}(t, a)|>|\operatorname{perm}(t, b)| \\
b \text { if }|\operatorname{perm}(t, b)|>|\operatorname{perm}(t, a)|
\end{array}\right.
$$

and $f$ is defined for every $t \in A$.

LemMa 5. $f$ is in $M$ and $G_{S_{0}}$ fixes $f$.

Proof. Let $t=\{a, b\} \in A$ and let $\psi^{\prime}$ be any element of $G_{s_{0}}$. Define $\psi$ as in the proof of Lemma 4. Then as in the proof of Lemma 4, $\psi(t)=\psi^{\prime}(t)$ and further $\psi(a)=\psi^{\prime}(a)$. (If not then $\psi^{-1} \psi^{\prime}(a)=b$ while $\psi^{-1} \psi^{\prime}(\{a, b\})=\{a, b\}$ contradicting the fact from Lemma 3 that $\left(\psi^{-1} \psi^{\prime}\right)^{3}=1_{U}$.) So that

$$
\begin{aligned}
|\operatorname{perm}(t, a)| & =|\{\eta \in \operatorname{perm}(t): g(\eta(t))=\eta(a)\}| \\
& =|\{\eta \psi \in \operatorname{perm}(t): g(\eta \psi(t))=\eta \psi(a)\}| \\
& =|\{\eta \in \operatorname{perm}(t): g(\eta(\psi(t)))=\eta \psi(a)\}| \\
& =\left|\operatorname{perm}\left(\psi^{\prime}(t), \psi^{\prime}(a)\right)\right| .
\end{aligned}
$$


Similarly $|\operatorname{perm}(t, b)|=\left|\operatorname{perm}\left(\psi^{\prime}(t), \psi^{\prime}(b)\right)\right|$. Therefore by the definition of $f$,

$$
f(t)=a \leftrightarrow f\left(\psi^{\prime}(t)\right)=\psi^{\prime}(a)
$$

proving Lemma 5 .

Now the proof of Theorem 3.2 is completed by proving the following: Claim. $f$ is $R$ consistent.

The proof is by contradiction. Suppose $t_{1}=\{a, b\}$ and $t_{2}=\{c, d\}$ are in $A$, that $f\left(t_{1}\right)=a, f\left(t_{2}\right)=c$ and further that $a R c$ is false.

Lemma 6. Suppose that $\eta \in \operatorname{perm}\left(\sup \left(t_{1}\right) \cap \sup \left(t_{2}\right)\right)$ and suppose that for some $\psi \in \operatorname{perm}\left(\sup \left(t_{1}\right)-\sup \left(t_{2}\right)\right), \quad \psi \eta \in \operatorname{perm}\left(t_{1}, a\right)$, i.e., $g\left(\psi \eta\left(t_{1}\right)\right)=\psi \eta(a)$. Then for every $\varphi \in \operatorname{perm}\left(\sup \left(t_{2}\right)-\sup \left(t_{1}\right)\right), \varphi \eta \in \operatorname{perm}\left(t_{2}, d\right)$, i.e., $g\left(\varphi \eta\left(t_{2}\right)\right)$ $=\varphi \eta(d)$.

Proof. If not then for some $\varphi \operatorname{perm}\left(\sup \left(t_{2}\right)-\sup \left(t_{1}\right)\right), \varphi \eta \in \operatorname{perm}\left(t_{2}, c\right)$ which means $g\left(\varphi \eta\left(t_{2}\right)\right)=\varphi \eta(c)$. Since $g$ is $R$-consistent we have $\psi \eta(a) R \varphi \eta(c)$. Since $R$ is fixed by $G_{S_{0}}$, we get

$$
\eta^{-1} \psi^{-1} \varphi^{-1} \psi \eta(a) R \eta^{-1} \psi^{-1} \varphi^{-1} \varphi \eta(c)
$$

Since $G$ is commutative we have $\varphi^{-1}(a) R \psi^{-1}(c)$. But $\psi \in \operatorname{perm}\left(\sup \left(t_{1}\right)-\right.$ $\left.-\sup \left(t_{2}\right)\right)$ and therefore, $\psi(c)=\psi^{-1}(c)=c$. Similarly $\varphi^{-1}(a)=a$ so we conclude that $a R c$, a contradiction. This completes the proof of Lemma 6.

We therefore conclude that for every $\eta \in \operatorname{perm}\left(\sup \left(t_{1}\right) \cap \sup \left(t_{2}\right)\right)$ either:

$$
\left(\forall \psi \in \operatorname{perm}\left(\sup \left(t_{1}\right)-\sup \left(t_{2}\right)\right)\right)\left(\psi \eta \in \operatorname{perm}\left(t_{1}, b\right)\right)
$$

or

Therefore if

$$
\left(\forall \varphi \in \operatorname{perm}\left(\sup \left(t_{2}\right)-\sup \left(t_{1}\right)\right)\right)\left(\varphi \eta \in \operatorname{perm}\left(t_{2}, d\right)\right) .
$$

and

$$
D_{1}=\mid\left\{\eta \in \operatorname{perm}\left(\sup \left(t_{1}\right) \cap \sup \left(t_{2}\right)\right):(1) \text { holds }\right\} \mid
$$

$$
D_{2}=\mid\left\{\eta \in \operatorname{perm}\left(\sup \left(t_{1}\right) \cap \sup \left(t_{2}\right)\right):(2) \text { holds }\right\} \mid,
$$

then $D_{1}+D_{2} \geqslant\left|\operatorname{perm}\left(\sup \left(t_{1}\right) \cap \sup \left(t_{2}\right)\right)\right|$. So either

$2 \cdot D_{1} \geqslant\left|\operatorname{perm}\left(\sup \left(t_{1}\right) \cap \sup \left(t_{2}\right)\right)\right| \quad$ or $\quad 2 \cdot D_{2} \geqslant\left|\operatorname{perm}\left(\sup \left(t_{1}\right) \cap \sup \left(t_{2}\right)\right)\right|$.

In the first case we would have $\left|\operatorname{perm}\left(t_{1}, b\right)\right|>\left|\operatorname{perm}\left(t_{1}, a\right)\right|\left(\right.$ since perm $\left(t_{1}\right)$ $=\operatorname{perm}\left(\sup \left(t_{1}\right)\right)$ can be written as perm $\left(\sup \left(t_{1}\right)\right)=\left\{\psi \eta: \psi \in \operatorname{perm}\left(\sup \left(t_{1}\right)-\right.\right.$ $\left.-\sup \left(t_{2}\right)\right)$ and $\left.\left.\eta \in \operatorname{perm}\left(\sup \left(t_{1}\right) \cap \sup \left(t_{2}\right)\right)\right\}\right)$ and this contradicts $f\left(t_{1}\right)=a$. Similarly in the second case we would have $\left|\operatorname{perm}\left(t_{2}, d\right)\right|>\left|\operatorname{perm}\left(t_{2}, c\right)\right|$ contradicting $f\left(t_{2}\right)=c$. This proves the claim and therefore Theorem 3.2.

\section{References}

[1] R. H. Cowen, Some combinatorial theorems equivalent to the prime ideal theorem, Proc. Amer. Math. Soc. 41 (1973), pp. 268-273.

[2] - Generalizing Konig's infinity lemma, Notre Dame J. Formal Logic 18 (1977), pp. 243-247. [3] - Binary consistent choice on triples, Notre Dame J. Formal Logic 18 (1977), pp. 310-312.

[3] - Binary consistent choice on triples, Notre Dame J. Formal The axiom of choice (Studies in logic and the foundations of mathematics, vol. 75), North-Holland, 1973.

[5] J. Łos and C. Ryl1-Nardzewski, On the application of Tychonoff's theorem in mathematical proofs, Fund. Math. 38 (1951), pp. 233-237.

[6] - Effectiveness of the representation theory for Boolean algebras, Fund. Math. 41 (1955), pp. 49-56.

Received 17 February 1981 